Ann. Biol. anim. Bioch. Biophys., r968, 8 (I), 87-93.

\title{
MODE D'ACTION DE L'ISORIBOFLAVINE SUR L'UTILISATION DE LA VITAMINE B2 PAR LA POULE PONDEUSE
}

\author{
J.-C. BLUM \\ Station de Recherches avicoles, \\ Centre national de Recherches zootechniques, 78 -Jouy-en-Josas \\ Institut national de la Recherche agronomique \\ Centre de Recherches sur la Nutrition, \\ ('entre national de la Recherche scientifique, 92 - Bellevue
}

\section{SOMMAIRE}

En se substituant à la riboflavine qui est transportée dans le sang sous forme d'une flavoprotéine, l'isoriboflavine rend une partie de la vitamine $B_{2}$ inutilisable pour le transfert à l'œuf. L'effet compétitif de l'antivitamine ne s'exercerait pas au niveau enzymatique, mais exclusivement au niveau de la forme de transport plasmatique.

\section{IN'TRODUCTION}

L'isoriboflavine 5,6 diméthyl-9 ( $\mathrm{I}^{\prime}$ D-ribityl) isoalloxazine est un antagoniste de la riboflavine chez le rat en croissance EMERSON et TISHL,ER, (I944). Dans le cadre d'une étude portant sur les mécanismes responsables du transfert de la riboflavine à l'œuf, nous avons voulu savoir si l'isoriboflavine a également un effet nocif chez la poule pondeuse et en particulier, dans quelle mesure elle s'oppose à l'incorporation de la vitamine à l'œuf.

Nous avons comparé les effets de 1'isoriboflavine à ceux précédemment étudiés d'une carence vitaminique BLUM, ( 1962 ). Habituellement, les antivitamines sont utilisées pour intensifier l'action d'un régime déficient. Nous avons abordé le problème d'une manière différente voulant savoir si en régime normal, l'isoriboflavine pouvait gêner préférentiellement l'incorporation de la vitamine dans l'œuf ou dans les tissus de la poule. 


\section{MATÉRIEL ET MÉ,THODES}

\section{a) Animaux et régimes}

Nous avons utilisé comme matériel expérimental des poules $R$ hode $\times$ Wyandotte choisies au cours de leur première année de ponte. Eau de boisson et aliments sont distribués ad libitum. Le régime utilisé à base de céréales, tourteaux divers et farine de poisson est complet et équilibré. Toutefois l'apport de riboflavine $(3 \mathrm{mg} / \mathrm{kg})$ est marginal ; il permet cependant d'assurer une bonne ponte et une teneur convenable de l'cuf en vitamine $\mathrm{B}_{2}$.

Au cours d'une prépériode de I mois ( 5 mai-I 5 juin), on récolte les œufs de 18 poules et on dose leur contenu en riboflavine (vitellus et albumen séparés). Puis les animaux sont répartis en 3 lots de telle sorte que le poids des oufs et l'intensité de ponte soient sensiblement identiques dans tous les lots.

- Le lot témoin continue à recevoir le régime de base ; il comprend 8 poules.

- Le lot I.200 reçoit le même régime de base additionné de $200 \mathrm{mg}$ d'isoriboflavine par $\mathrm{kg}$; il ne comprend que 4 animaux. 6 animaux.

- Le lot I.500 reçoit le régime de base additionné de $500 \mathrm{mg}$ d'isoriboflavine par $\mathrm{kg}$; il comprend

L'expérience proprement dite débute le $I_{5}$ juin. On sacrifie : le 17 juin (après 48 heures d'expérience) deux animaux du lot témoin et deux animaux du lot I.500, le I er juillet ( 5 jours d'expérience) deux animaux de chaque lot, le 8 juillet ( 3 semaines d'expérience) deux animaux de chaque lot, le 12 juillet les deux témoins restants.

La riboflavine est dosée dans tous les oufs pondus. De ce fait, on dispose pour chaque animal d'un grand nombre de résultats (cf. indice de ponte : tabl. I) montrant l'évolution du contenu vitaminique de l'œuf en cours d'expérience.

\section{b) Méthode de dosage}

Nous avons dosé la vitamine $B_{2}$ par voie microbiologique selon la méthode de SNELL et STRong (1939) qui utilise Lactobacillus casei. ForsTER (1944) a montré que l'isoriboflavine n'entrait pas en compétition avec la vitamine $B_{2}$ pour inhiber le développement de Lactobacillus casei. Cependant, selon cet auteur, l'isoriboflavine aurait une faible activité vitaminique qu'il qualifie de négligeable: inférieure à $0,5 \mathrm{p}$. Ioo de celle de la riboflavine.

Nous avons testé l'effet de l'isoriboflavine sur la croissance de Lactobacillus casei. Quelle que soit la dose introduite (entre o et $5 \circ \mu \mathrm{g}$ par ml de milieu) cette substance s'est révélée dépourvue de toute activité vitaminique. La croissance du micro-organisme ne dépend que de la quantité de riboflavine introduite ; elle n'est ni gênée ni exaltée par l'isoriboflavine.

En bref, l'isoriboflavine n'est pour Lactobacillus casei ni une vitamine, ni une antivitamine ; ce qui assure la validité des dosages en présence de cet antagoniste. Quant à la signification biologique de cette indifférence du lactobacille, nous l'envisagerons ultérieurement en discutant de l'effet de l'isoriboflavine chez la poule pondeuse.

\section{RÉSULTATS}

\section{a) Intensité de ponte}

L'indice de ponte (nombre d'œufs pondus en un jour par cent poules) et son évolution en cours d'expérience sont indiqués dans le tableau $x$. Les périodes ont été délimitées par les dates de mise en expérience et de sacrifices.

L'intensité de ponte est élevée dans tous les lots ; elle n'est pas diminuée par l'administration d'isoriboflavine. 
b) Analyse du vitellus et de l'albumen des ceuts pondus

L'évolution de la teneur moyenne en riboflavine ( $\mu$ g par $\mathrm{g}$ frais) de chacun des constituants de l'œuf est indiquée dans le tableau 2.

TABLEAU I

Évolution de l'indice de ponte en cours d'expérience

\begin{tabular}{|c|c|c|c|c|}
\hline & $\begin{array}{c}\text { Prépériode } \\
\text { (15 mai-15 juin) }\end{array}$ & 16 juin et 17 juin & 18 juin-1er juillet & 2 juillet- 8 juillet \\
\hline $\begin{array}{l}\text { Lot témoin } \ldots \ldots \ldots \ldots \ldots \\
\text { Lot } I .200 \ldots \ldots \ldots \ldots \ldots \\
\text { Lot } I .500 \ldots \ldots \ldots \ldots \ldots\end{array}$ & $\begin{array}{l}72 \\
75 \\
75\end{array}$ & $\begin{array}{l}75 \\
83 \\
83\end{array}$ & $\begin{array}{l}67 \\
73 \\
73\end{array}$ & $\begin{array}{l}67 \\
80 \\
82\end{array}$ \\
\hline
\end{tabular}

TABLEAU 2

Teneurs moyennes en riboflavine ( $\mu \mathrm{g}$ par $\mathrm{g}$ frais) du vitellus et de l'albumen de l'wuf pondu

\begin{tabular}{|c|c|c|c|c|}
\hline & $\begin{array}{c}\text { Prépériode } \\
\text { (15 mai-15 juin) }\end{array}$ & 16 juin et 17 juin & 18 juin-1er juillet & 2 juillet-8 juillet \\
\hline \multicolumn{5}{|l|}{ Vitellus } \\
\hline Lot témoin ..... & 3,3 & 3,5 & 3,1 & 3,3 \\
\hline Lot $1.200 \ldots \ldots \ldots$ & 2,9 & 2,4 & 2,3 & 2,3 \\
\hline Lot I.500 $\ldots \ldots \ldots \ldots \ldots$ & 3,5 & 3,3 & 2,1 & 1,7 \\
\hline \multicolumn{5}{|l|}{ Albumen } \\
\hline Lot témoin $\ldots \ldots \ldots \ldots \ldots$ & 2,2 & 2,4 & 2,1 & 2,5 \\
\hline Lot $1.200 \ldots \ldots \ldots \ldots \ldots$ & 2,4 & 2,5 & 2,2 & 2,2 \\
\hline Lot $1.500 \ldots \ldots \ldots \ldots$ & 2,4 & 2,4 & 1,6 & 1,5 \\
\hline
\end{tabular}

L'influence du facteur individuel se fait particulièrement sentir dans les dernières périodes en raison du nombre faible des animaux restant après sacrifice. Pour l'éliminer on utilise un artifice de calcul : les valeurs de la prépériode sont affectées pour chaque poule du coefficient Ioo. La teneur du vitellus et de l'albumen des ceufs suivants s'exprime alors en pourcentage de la prépériode. C'est la moyenne de ces pourcentages qui figure dans le tableau 3 .

De ces deux tableaux, il ressort qu'en fin d'expérience :

- l'adjonction de $200 \mathrm{mg}$ d'isoriboflavine par $\mathrm{kg}$ de régime a provoqué une diminution sensible de la teneur vitaminique du vitellus ( 20 à $30 \mathrm{p}$. roo), tandis que celle de l'albumen est peu modifiée (baisse d'environ ro p. IOo) ;

- l'adjonction de $500 \mathrm{mg}$ d'isoriboflavine a diminué d'environ $50 \mathrm{p}$. Ioo la concentration vitaminique du jaune d'œuf, tandis que le blanc s'est moins appauvri $(-37$ p. I00) ; 
- dans le lot I.50o, le contenu en vitamine du vitellus diminue progressivement et n'atteint une valeur minimum qu'après une dizaine de jours ; 1'albumen conserve sa richesse initiale pendant 48 heures environ, sa teneur diminue alors brutalement jusqu'à une valeur qui reste la même jusqu'à la fin de l'expérience.

TABLEAU 3

Évolution de la teneur en riboflavine du vitellus et de l'albumen

à partir de la première période considérée comme égale à 100

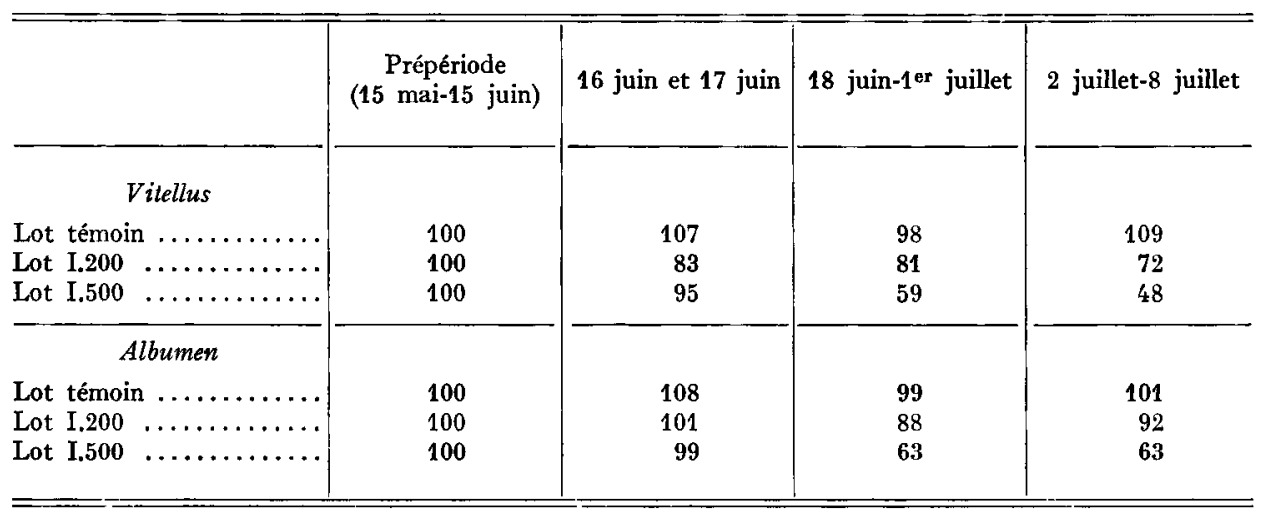

c) Analyse du foie

Dans le tableau 4, nous indiquons la teneur en riboflavine en $\mu \mathrm{g}$ par gramme frais et également en $\mu \mathrm{g}$ par gramme d'azote (dosée par macrokjeldahl). A titre d'indication, nous mentionnons aussi le poids du foie. Pour le lot témoin, nous avons fait figurer ces valeurs sous forme de moyennes en indiquant l'écart-type pour donner une idée de la variabilité; pour les autres lots afin de rechercher l'influence éventuelle de la durée d'administration du régime, nous donnons les résultats individuels.

La teneur en riboflavine diffère d'un individu à l'autre; elle paraît cependant moins variable lorsque l'azote est utilisé comme paramètre de référence. En ce cas, toutes les valeurs obtenues dans les lots I.200 et I.500 sont inférieures à la moyenne des témoins. La différence reste cependant faible et, du fait de la variabilité et du nombre peu élevé d'animaux, cette différence n'est pas significative.

\section{DISCUSSION}

Les résultats obtenus tendent à montrer qu'il n'y a pas compétition entre la vitamine $B_{2}$ et l'isoriboflavine au niveau dư foie de la poule. Ce caractère est à rapprocher de l'indifférence de Lactobacillus casei à l'égard de l'antivitamine $\mathbf{B}_{\mathbf{2}}$ (Cf. Matériel et méthodes). On est en droit de penser que l'isoriboflavine n'entre pas en compétition avec la vitamine $B_{2}$ pour constituer les flavoprotéines-enzymes qui sont indispensables pour le lactobacille et qui constituent la majeure partie de la ribo- 
MODE D'ACTION DE L'ISORIBOFLAVINE

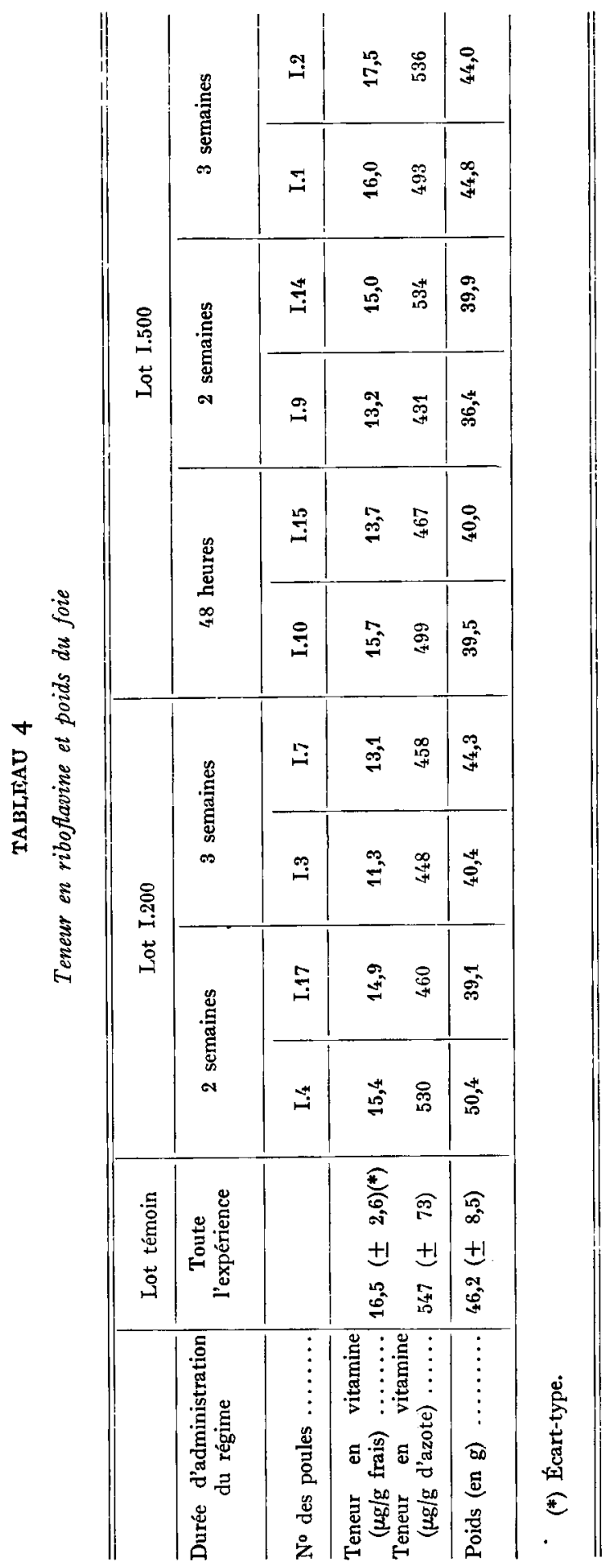


flavine hépatique. Cette hypothèse trouve confirmation dans les résultats obtenus par divers auteurs qui montrent que ni l'isoriboflavine, ni une autre antivitamine $B_{2}, 1$ galactoflavine, ne peuvent servir de substrats artificiels pour la synthèse de nucléotides flaviniques (MCCoRmick, I96I ; MCCormCK et BuTLER, I962 ; Prosky et al. Ig64).

Au cours de travaux antérieurs (BI,UM et JACQUOT-ARMAND, I965; BLUM, I966), nous avons vu chez la poule pondeuse que la riboflavine est transportée par le sang et introduite dans l'œuf sous forme de flavoprotéines. $\mathrm{L}_{1}$ a vitamine $\mathrm{B}_{2}$ de ces flavoprotéines n'est pas sous forme de nucléotides et in vitro elle est déplacée partiellement par l'isoriboflavine. Cette substitution ayant lieu à la température de $+4^{\circ}$ semble n'exiger aucune réaction enzymatique. On peut supposer que l'isoriboflavine a le même effet in vivo. Ainsi s'explique son action sur le transfert de la vitamine à l'œuf. En déplaçant la riboflavine de son support plasmatique, l'isoriboflavine induit l'excrétion de la vitamine et la rend inutilisable pour le transfert à l'œuf. Cette influence s'exerce dès le jour où l'isoriboflavine est ajoutée au régime et son intensité dépend des proportions relatives de riboflavine et d'antivitamine. S'il faut un certain temps de latence pour qu'elle se manifeste pleinement au niveau de chacun des constituants de l'œuf, c'est parce que le dépôt de la vitamine s'y effectue progressivement suivant une cinétique que nous avons analysée par ailleurs BLUM, (I965).

\section{CONCLUSION}

Chez la poule pondeuse, il y a compétition entre la vitamine $B_{2}$ et l'isoriboflavine pour la forme de transport plasmatique, en revanche cette compétition n'existe pas au niveau enzymatique. L'action de l'antivitamine n'est marquée que lorsque le rôle de l'apport plasmatique est essentiel ; ce qui est le cas pour la formation du vitellus, et à un moindre titre, de 1'albumen. On dissocie ainsi deux modes d'intervention de la vitamine $B_{2}$ dans la vitellogenèse, d'une part en tant que constituant de l'œuf et d'autre part comme biocatalyseur essentiel dans le métabolisme intermédiaire. Lorsque le régime apporte suffisamment de vitamine $B_{2}$, l'isoriboflavine ne gêne ni la ponte, ni aucune fonction physiologique; mais en appauvrissant 1'œuf en riboflavine, elle compromet à coup sûr le développement embryonnaire. Ainsi une substance d'origine alimentaire pourra jouer le rôle d'antivitamine exclusivement pour l'embryon et non pour l'organisme maternel.

Rę̧u pour publication en novembre 1967.

\section{SUMMARY}

MODE OF ACTION OF ISORIBOFLAVINE ON THE UTILIZATION OF VITAMIN $B_{2}$ BY LAYING HENS

A large amount of isoriboflavine (lot $\mathrm{I} 500=500 \mathrm{mg}$ per $\mathrm{kg}$ feed) was given to laying hens in a complete balanced diet containing $3 \mathrm{mg}$ vitamin $B_{2}$ per $\mathrm{kg}$. There was a reduction in riboflavine content of the egg ; the content of the vitamin diminished in yolk by a half and in white by a third. There was no effect on reserves of riboflavine in liver. 
In a previous study (BLUM, I966) we showed the presence in blood of a flavoprotein which assured the transport of riboflavine and its incorporation into the egg. The riboflavine of this flavoprotein can be replaced in vitro by isoriboflavine. It is doubtlesse the same in vivo and part of the vitamin $B_{2}$ then becomes unusable for transfer to the egg. The constancy of the reserves in liver confirms other work, and permits the supposition that isoriboflavine does not compete with riboflavine in the formation of enzyme flavoproteins in tissues.

\section{RÉFÉRENCES BIBLIOGRAPHIQUES}

BLUM J.- C., 1965. Utilisation de la riboflavine radioactive pour l'étude du métabolisme de la vitamine $B_{2}$ au cours de l'ovogenèse chez la poule pondeuse. Intern. Symp.C.A.M.I. R.A., Meded. Landbourohogeschool Gent., 30, $78 \mathrm{r}-786$.

BLum J.-C., I966. Les flavoprotéines de l'ouf et du sérum sanguin de la poule pondeuse. C. R. Acad. Sci., 262, I299-I 30I.

BLUM J.-C., JACQUOT-ARMAND Y., 1965. Le métabolisme de la riboflavine au cours de l'ovogenèse chez la poule pondeuse. Ann. Nutr. Alim., 19, C599-C610.

EmERSon G. A., TISHLER M., r944. The antiriboflavin effect of isoriboflavin. Proc. Soc. exp. Biol. Med, $55,184-185$.

FORSTER J. W., I944. Microbiological aspects of riboflavin. J. Bacteriol., 48, 97-1 I r.

MccoRMICK D. B., I96I. The intracellular localization, partial purification and properties of flavokinase, from rat liver. J. Biol. Chem., 23\%, 959-962.

Mccormick D. B., BUTLER R. C., I962. Sustrate specificity of liver flavokinase. Biochim. Biophys. Acla., 65, 326-332.

Prosky L., Burch H. B., Bejrablaya D., Lowry O. H., Combs A. M., 1964. The effects of galactoflavine on riboflovin enzymes and coenzymes. J. Biol. Chem., 239, $269 \mathrm{I}-2695$.

SNELl E. E., Strong F. M., 1939. A microbiological assay for riboflavin. Industr. Eng. Chem. Anal. Ed. 11, 346-350. 